\title{
A DELAY LOGISTIC EQUATION WITH VARIABLE GROWTH RATE*
}

\author{
DONALD S. COHEN $\dagger$ AND S. ROSENBLAT $\$$
}

\begin{abstract}
A logistic equation with distributed delay is considered in the case where the growth rate oscillates sinusoidally about a positive mean value. A delay kernel is chosen which admits bifurcation of the equilibrium state into a periodic solution when the growth rate is constant. It is shown that the fluctuations in growth rate modulate the bifurcation into a quasiperiodic solution. In certain circumstances, however, it is shown that frequency locking can occur but that this is a local phenomenon which does not persist outside the immediate vicinity of the bifurcation point.
\end{abstract}

1. Introduction. J. M. Cushing [1] has investigated stability and bifurcation for a Volterra delay logistic equation of the form

$$
U_{t} / U=\alpha\left[1-\beta \int_{-\infty}^{t} K\left(t-t^{\prime}\right) U\left(t^{\prime}\right) d t^{\prime}\right]
$$

where $U$ is the population density of the species at time $t, \alpha$ is the growth rate, $\beta$ is a positive constant $\left(\beta^{-1}\right.$ is the carrying capacity) and $K$ is a delay kernel. Without loss of generality it may be assumed that $K$ is normalized in such a way that

$$
\int_{0}^{\infty} K\left(t^{\prime}\right) d t^{\prime}=\int_{-\infty}^{t} K\left(t-t^{\prime}\right) d t^{\prime}=1
$$

When (1.2) holds, (1.1) admits the positive equilibrium solution

$$
U(t) \equiv \beta^{-1} \text {. }
$$

If $T$ is the characteristic time associated with the delay kernel $K$, it is possible to redefine $t$ as a dimensionless time scaled on $T$, so that in the new variable the delay time is unity. Under this transformation (1.1) remains unchanged, except that now $\alpha$ is a dimensionless growth rate parameter which measures the ratio of delay time to growth time. We assume that (1.1) is dimensionless in time in this sense.

Cushing [1] studied the stability of the solution (1.3), giving details particularly in the case of the delay kernel

$$
K(t)=t e^{-t} \text {. }
$$

He showed that when (1.4) applies the solution (1.3) is asymptotically stable for $\alpha<2$ and unstable for $\alpha>2$. In addition Cushing demonstrated the occurrence of a Hopf bifurcation at the critical value $\alpha=2$ and the appearance of a stable periodic bifurcating solution for $\alpha>2$.

In this paper we propose to examine the behavior of (1.1) when the growth rate, instead of being constant, varies periodically about a constant positive mean value. This can be regarded as modeling cyclical fluctuations caused, for example, by seasonal variation in the availability of nutrient.

We shall suppose that the amplitude of the variations is small relative to the mean growth rate. In this case, as one might expect, the response of the system is small except in the neighborhood of a critical point, which means, in the present context, close to the bifurcation point mentioned above. Hence we restrict our analysis

\footnotetext{
* Received by the editors April 22, 1980, and in revised form March 27, 1981.

$\dagger$ Department of Applied Mathematics, California Institute of Technology, Pasadena, California 91125.

$\ddagger$ Department of Mathematics, Illinois Institute of Technology, Chicago, Illinois 60616 .
} 
to the neighborhood of the bifurcation point where, as we shall see, the effect of even a small fluctuation in the growth rate can be qualitatively significant.

Specifically we consider in place of (1.1) the equation

$$
\frac{U_{t}}{U}=\alpha(t)\left[1-\beta \int_{-\infty}^{t} K\left(t-t^{\prime}\right) U\left(t^{\prime}\right) d t^{\prime}\right]
$$

where

$$
\alpha(t) \equiv \alpha[1+\varepsilon \cos \Omega t]
$$

with $0<\varepsilon \ll 1$ and $\Omega>0$. The frequency $\Omega$ effectively constitutes an additional time scale for the problem and plays an important role in the qualitative behavior of the system.

In this paper we shall limit the discussion to the case where the delay kernel $K$ has the form (1.4). This allows considerable simplification of the algebra while retaining the principal qualitative features of the general case.

In $\S 2$ we present the stability and bifurcation discussion for (1.5)-(1.6), using a perturbation analysis based on the smallness of the parameter $\varepsilon$. The results so obtained are found to be valid except when

$$
\Omega \approx 1,2 \text { or } 3 \text {; }
$$

the general analysis breaks down at the resonance values (1.7) of the forcing frequency. We find that the location of the bifurcation point is displaced a distance $O\left(\varepsilon^{2}\right)$ and that the norm of the bifurcating solution is $O(\varepsilon)$. The bifurcating solution experiences a frequency shift at leading order but, in reality, is modulated into a quasiperiodic solution as a consequence of the growth rate fluctuation.

The resonance cases (1.7) are considered in $\S \S 3-5$ respectively. When $\Omega$ is sufficiently close to 1 we find that the bifurcating solution is periodic with frequency $\Omega$, and has norm $O(\varepsilon)$. We show, however, that this phenomenon of frequency locking is purely local: the locked-in periodic bifurcating solution does not persist beyond a neighborhood of the bifurcation point. We conjecture, but do not prove, that it is replaced by a solution of quasiperiodic type outside its neighborhood of existence.

Very similar behavior is found when $\Omega$ is very close to 2 , except that in this (subharmonic) case the locked-in frequency is $\frac{1}{2} \Omega$ and the norm of the bifurcating solution is $O\left(\varepsilon^{1 / 2}\right)$. The response is thus greatest when $\Omega \approx 2$. Once again the frequency locking does not persist outside a neighborhood of the bifurcation point.

When $\Omega \approx 3$ we find that frequency locking occurs only at one special value of $\Omega$, and even then it is again a purely local phenomenon. In general when $\Omega \approx 3$ the solution has essentially the behavior appropriate to the nonresonance situation described in $\S 2$.

2. Bifurcation: general case. We consider the system (1.5)-(1.6) with delay kernel defined by (1.4). We set

$$
U(t)=\beta^{-1}+u(t)
$$

whereupon (1.5) takes the form

$$
u_{t}=-\alpha(t) \int_{-\infty}^{t} K\left(t-t^{\prime}\right) u\left(t^{\prime}\right) d t^{\prime}-\alpha(t) \beta u \int_{-\infty}^{t} K\left(t-t^{\prime}\right) u\left(t^{\prime}\right) d t^{\prime}
$$

This equation admits the equilibrium solution $u(t) \equiv 0$. 
For the kernel (1.4) it is well known that (2.2) can be replaced by an equivalent system of three ordinary differential equations. We define

$$
v(t)=\int_{-\infty}^{t}\left(t-t^{\prime}\right) e^{-\left(t-t^{\prime}\right)} u\left(t^{\prime}\right) d t^{\prime}, \quad w(t)=\int_{-\infty}^{t} e^{-\left(t-t^{\prime}\right)} u\left(t^{\prime}\right) d t^{\prime}
$$

and obtain in place of (2.2) the system

$$
\begin{aligned}
& u_{t}=-\alpha(1+\varepsilon \cos \Omega t)(v+\beta u v), \\
& v_{t}=-v+w, \quad w_{t}=u-w .
\end{aligned}
$$

In this section we discuss the bifurcation problem for arbitrary values of $\Omega$, using a perturbation procedure based on the small parameter $\varepsilon$.

Before proceeding we note what happens when $\varepsilon=0$. To determine the stability of the null solution when $\varepsilon=0$ we omit the nonlinear term in (2.4) and set $u, v, w$ proportional to $\exp (\nu t)$. This leads to the characteristic equation

$$
\nu(\nu+1)^{2}+\alpha=0 ;
$$

it is easily verified that (2.5) has a pair of complex conjugate roots whose real part changes from negative to positive as $\alpha$ increases through the value 2. This fixes $\alpha=2$ as the critical point; the roots of $(2.5)$ at criticality are

$$
\nu= \pm i \text { and } \nu=-2 \text {, }
$$

so that the natural frequency is 1 .

Next, a standard analysis reveals that a nontrivial periodic solution bifurcates from the null solution at the point $\alpha=2$. This solution is supercritical (exists for $\alpha>2$ ) and, by virtue of Hopf bifurcation theory, is stable. A straight forward calculation shows that the bifurcating solution when $\varepsilon=0$ has the form

$$
u(t)=\frac{2 \sqrt{2(\alpha-2)}}{\beta} \cos \left[t+\frac{1}{6}(\alpha-2) t\right]+O(\alpha-2),
$$

with corresponding expressions for $v$ and $w$, when $|\alpha-2|$ is small and $\alpha>2$. As can be seen from (2.7), the amplitude is proportional to $\sqrt{(\alpha-2)}$ and the frequency shift is proportional to $\alpha-2$.

We return now to the general problem with $\varepsilon \neq 0$. We use a multiple scaling technique, with fast time $t$ and slow time $\tau$ defined by

$$
\tau=\varepsilon^{2} t \text {. }
$$

In standard fashion the system (2.4) then becomes

$$
\begin{aligned}
& u_{t}+\varepsilon^{2} u_{\tau}=-\alpha(1+\varepsilon \cos \Omega t)(v+\beta u v), \\
& v_{t}+\varepsilon^{2} v_{\tau}=-v+w, \quad w_{t}+\varepsilon^{2} w_{\tau}=u-w .
\end{aligned}
$$

The vector $\mathbf{q}=(u, v, w)$ is now expanded in the form

$$
\mathbf{q}(t, \tau)=\varepsilon \mathbf{q}_{1}(t, \tau)+\varepsilon^{2} \mathbf{q}_{2}(t, \tau)+\varepsilon^{3} \mathbf{q}_{3}(t, \tau)+\cdots,
$$

and we set

$$
\alpha=2\left(1+\varepsilon^{2} \mu+\cdots\right),
$$

where $\mu$ is a constant. In these representations the parameter $\varepsilon$ measures the norm of the bifurcating solution. 
Substituting (2.10)-(2.11) into (2.9) and equating coefficients of like powers of $\varepsilon$ we obtain the following sequence of equations:

$$
\begin{aligned}
& L\left(\mathbf{q}_{1}\right)=(0,0,0), \\
& L\left(\mathbf{q}_{2}\right)=\left(-2 \beta u_{1} v_{1}-2 \cos \Omega t v_{1}, 0,0\right), \\
& L\left(\mathbf{q}_{3}\right)=-\left(u_{1, \tau}+2 \mu v_{1}+Z, v_{1, \tau}, w_{1, \tau}\right),
\end{aligned}
$$

where $L$ is the matrix differential operator defined by

$$
L=\left[\begin{array}{ccc}
\frac{\partial}{\partial t} & 2 & 0 \\
0 & \frac{\partial}{\partial t}+1 & -1 \\
-1 & 0 & \frac{\partial}{\partial t}+1
\end{array}\right]
$$

and $Z$ is defined by

$$
Z=2\left[\cos \Omega t v_{2}+\beta\left(u_{1} v_{2}+u_{2} v_{1}\right)+\beta \cos \Omega t u_{1} v_{1}\right]
$$

The general (periodic) solution of (2.12) is

$$
\begin{aligned}
u_{1}(t, \tau) & =2 A(\tau) \cos t+2 B(\tau) \sin t, \\
v_{1}(t, \tau) & =A(\tau) \sin t-B(\tau) \cos t, \\
w_{1}(t, \tau) & =A(\tau)(\cos t+\sin t)+B(\tau)(\sin t-\cos t),
\end{aligned}
$$

where $A, B$ are arbitrary functions of $\tau$. The solution of (2.13) can be written in the form

$$
\mathbf{q}_{2}=\hat{\mathbf{q}}_{2}+\mathbf{Q}_{2}
$$

where

$$
L\left(\hat{\mathbf{q}}_{2}\right)=\left(-2 \beta u_{1} v_{1}, 0,0\right)
$$

and

$$
L\left(\mathbf{Q}_{2}\right)=\left(-2 \cos \Omega t v_{1}, 0,0\right)
$$

Equation (2.19) arises in the calculations of the bifurcating solution when $\varepsilon=0$ and always has a periodic solution. Equation (2.20), on the other hand, is the contribution from the variable growth rate and is uniquely solvable in periodic functions provided that

$$
\Omega \neq 2 .
$$

If (2.21) does not hold there is a resonance situation which needs to be considered separately. We assume in the remainder of this section that $(2.21)$ holds; then the solution vector $\mathbf{Q}_{2}$ is evidently a linear combination of $\cos (\Omega \pm 1) t$ and $\sin (\Omega \pm 1) t$, and is linear in $A$ and $B$. After some tedious calculations we find that

$$
V_{2}=\sum_{i=1}^{2} \frac{1}{\left(1-\Lambda_{i}^{2}\right)\left(4+\Lambda_{i}^{2}\right)}\left\{\left(2 B+\Lambda_{i} A\right) \cos \Lambda_{i} t+\left(\Lambda_{i} B-2 A\right) \sin \Lambda_{i} t\right\},
$$

where $\Lambda_{1}=1+\Omega, \Lambda_{2}=1-\Omega$. The corresponding expressions for $U_{2}$ and $W_{2}$ are not explicitly required. 
We turn next to the system (2.14). Let $\bar{Z}$ denote the part of the quantity $Z$ defined by $(2.16)$ which is $2 \pi$-periodic in $t$. Then (2.14) has a unique bounded solution provided the vector

$$
\left(u_{1, \tau}+2 \mu v_{1}+\bar{Z}, v_{1, \tau}, w_{1, \tau}\right)
$$

is orthogonal to each of the $2 \pi$-periodic eigensolutions of the system adjoint to (2.12). The adjoint eigenvectors are easily shown to be

$$
(\cos t, \sin t-\cos t, \sin t), \quad(-\sin t, \sin t+\cos t, \cos t) .
$$

Then applying the orthogonality conditions we obtain, after some rearrangement, the following pair of equations for the amplitude functions $A$ and $B$ :

$$
\begin{aligned}
5 A_{\tau}-\mu A-2 \mu B+\langle\bar{Z}, 2 \cos t-\sin t\rangle & =0, \\
5 B_{\tau}+2 \mu A-\mu B+\langle\bar{Z}, \cos t+2 \sin t\rangle & =0,
\end{aligned}
$$

where $\langle\cdot, \cdot\rangle$ denotes inner product over the period $2 \pi$.

It is necessary now to make a closer examination of the terms involved in the expression for $Z$. For example, since both $u_{1}$ and $v_{1}$ are sinusoidal functions with period $2 \pi$, it is clear that the last term $\beta \cos \Omega t u_{1} v_{1}$ makes no contribution to $\bar{Z}$, and hence none to the inner products in (2.25), except in the special cases $\Omega=1$ and $\Omega=3$. Precisely the same is true of the terms $\cos \Omega t \hat{v}_{2}$ and $\beta\left(u_{1} V_{2}+U_{2} v_{1}\right)$. On the other hand, the terms $\cos \Omega t V_{2}$ and $\beta\left(u_{1} \hat{v}_{2}+\hat{u}_{2} v_{1}\right)$ make nontrivial contributions to the inner products for all values of $\Omega$. We assume now that

$$
\Omega \neq 1, \quad \Omega \neq 3 .
$$

Then (2.16) can be written in the form

$$
Z=2\left[\cos \Omega t V_{2}+\beta\left(u_{1} \hat{v}_{2}+\hat{u}_{2} v_{1}\right)\right]+\cdots
$$

and only the terms explicitly displayed make nonzero contributions to the inner products.

The required calculations are now easily performed. We find that when (2.21) and (2.26) hold the solvability conditions (2.25) for the amplitude functions become

$$
\begin{array}{r}
5 A_{\tau}+(-\mu+p) A+(-2 \mu+q) B+\frac{1}{12} \beta^{2} R^{2}(3 A+11 B)=0, \\
5 B_{\tau}+(2 \mu-q) A+(-\mu+p) B-\frac{1}{12} \beta^{2} R^{2}(11 A-3 B)=0,
\end{array}
$$

where

$$
\begin{aligned}
& p=\frac{1+\Lambda_{1}}{\left(1-\Lambda_{1}^{2}\right)\left(4+\Lambda_{1}^{2}\right)}+\frac{1+\Lambda_{2}}{\left(1-\Lambda_{2}^{2}\right)\left(4+\Lambda_{2}^{2}\right)}, \\
& q=\frac{4-\Lambda_{1}}{2\left(1-\Lambda_{1}^{2}\right)\left(4+\Lambda_{1}^{2}\right)}+\frac{4-\Lambda_{2}}{2\left(1-\Lambda_{2}^{2}\right)\left(4+\Lambda_{2}^{2}\right)},
\end{aligned}
$$

where $\Lambda_{1}, \Lambda_{2}$ are as defined above, and where $R^{2}=A^{2}+B^{2}$. In fact, defining polar coordinates $R, \theta$ by the formulas

$$
A=R \cos \theta, \quad B=R \sin \theta,
$$

we transform (2.28) into the equations

$$
5 R_{\tau}=(\mu-p) R-\frac{1}{4} \beta^{2} R^{3}, \quad 5 \theta_{\tau}=-2 \mu+q+\frac{11}{12} \beta^{2} R^{2} .
$$


The first of these shows that the null solution loses stability at the value $\mu=p$, which means, by virtue of $(2.11)$, at the value

$$
\alpha=2\left(1+\varepsilon^{2} p+\cdots\right) \text {. }
$$

Thus there is in general an $O\left(\varepsilon^{2}\right)$ displacement of the bifurcation point. As solutions of $(2.31)$ we obtain

$$
R=\frac{2 \sqrt{\mu-p}}{\beta}, \quad \theta=\left(\frac{1}{3} \mu+\frac{1}{5} q-\frac{11}{15} p\right) \tau
$$

for $\mu>p$.

Reverting to the original variables we find that (2.32) and (2.33) imply the solution

$$
u(t)=\frac{2 \sqrt{2\left(\alpha-2-\varepsilon^{2} p\right)}}{\beta} \cos \left\{t+\frac{1}{6}(\alpha-2) t+\left(\frac{1}{5} q-\frac{11}{15} p\right) \varepsilon^{2} t\right\}+O(\alpha-2)+O\left(\varepsilon^{2}\right)
$$

Comparing with (2.7) we see that the solution is periodic at leading order, but with a modified amplitude and frequency. However, the term represented by $O\left(\varepsilon^{2}\right)$ in (2.34) is the appropriate component of $\mathbf{Q}_{2}$, defined by (2.20), and the frequency is in general different from that of the leading order term. Hence we infer that the effect of the growth rate fluctuation is to modulate the periodic bifurcating solution into a quasiperiodic solution (which is supercritical and stable).

We now consider in turn the excluded special cases $\Omega=1, \Omega=2$ and $\Omega=3$.

3. Bifurcation when $\boldsymbol{\Omega} \approx \mathbf{1}$. It is of interest to consider what happens not only when $\Omega$ is exactly equal to 1 but also when $\Omega$ is close to 1 . To this end we study the situation where

$$
\Omega=1+\varepsilon^{2} \gamma
$$

with $\gamma$ a real constant.

The convenient time scales for this case are represented by the variables $t^{*}, \tau$ where

$$
t^{*}=\left(1+\varepsilon^{2} \gamma\right) t, \quad \tau=\varepsilon^{2} t
$$

as a result, the system (2.4) becomes

$$
\begin{aligned}
& \left(1+\varepsilon^{2} \gamma\right) u_{t^{*}}+\varepsilon^{2} u_{\tau}=-\alpha\left(1+\varepsilon \cos t^{*}\right)(v+\beta u v), \\
& \left(1+\varepsilon^{2} \gamma\right) v_{t^{*}}+\varepsilon^{2} v_{\tau}=-v+w, \\
& \left(1+\varepsilon^{2} \gamma\right) w_{t^{*}}+\varepsilon^{2} w_{\tau}=u-w .
\end{aligned}
$$

The vector $\mathbf{q}=(u, v, w)$ is expanded in a series of the form (2.10) and the bifurcation parameter $\mu$ is introduced through the representation (2.11). Substituting into (3.3) and equating coefficients of like powers of $\varepsilon$ we obtain a sequence of equations very similar to (2.12)-(2.14). The solution of the leading order equation, corresponding to (2.12), is precisely (2.17) except that $t$ is replaced by $t^{*}$. The solution of the analog of (2.13) can again be written in the form $q_{2}=\hat{\mathbf{q}}_{2}+Q_{2}$, with (2.19) and (2.20) the governing equations except that $t^{*}$ replaces $\Omega t$. In particular we find that

$$
\begin{aligned}
& U_{2}=\frac{1}{2} B+\frac{1}{12}(7 A-B) \cos 2 t^{*}+\frac{1}{12}(A+7 B) \sin 2 t^{*}, \\
& V_{2}=\frac{1}{2} B-\frac{1}{12}(A+B) \cos 2 t^{*}+\frac{1}{12}(A-B) \sin 2 t^{*},
\end{aligned}
$$

with a corresponding expression for $W_{2}$, which is not explicitly needed. 
At third order we find that

$$
L\left(\mathbf{q}_{3}\right)=-\left(u_{1, \tau}+\gamma u_{1, t^{*}}+2 \mu v_{1}+Z, v_{1, \tau}+\gamma v_{1, t^{*}}, w_{1, \tau}+\gamma w_{1, t^{*}}\right),
$$

where $Z$ is given by (2.16) with $\Omega t$ replaced by $t^{*}$. We apply the Fredholm alternative in the usual way to obtain the following solvability conditions, which are obvious generalizations of $(2.25)$ :

$$
\begin{aligned}
& 5 A_{\tau}-\mu A+(5 \gamma-2 \mu) B+\left\langle Z, 2 \cos t^{*}-\sin t^{*}\right\rangle=0, \\
& 5 B_{\tau}+(2 \mu-5 \gamma) A-\mu B+\left\langle Z, \cos t^{*}+2 \sin t^{*}\right\rangle=0 .
\end{aligned}
$$

As mentioned in $\S 2$, all the terms in $Z$ now contribute to the inner product. After some lengthy but straightforward algebra we obtain eventually the amplitude equations for $A$ and $B$ :

$$
\begin{aligned}
5 A_{\tau}-\left(\mu+\frac{1}{8}\right) A+\left(5 \gamma-2 \mu+\frac{23}{24}\right) B & -\frac{1}{24} \beta\left(15 A^{2}+27 B^{2}+16 A B\right) \\
& +\frac{1}{12} \beta^{2} R^{2}(3 A+11 B)=0 \\
5 B_{\tau}+\left(2 \mu-5 \gamma+\frac{1}{24}\right) A-\left(\mu-\frac{3}{8}\right) B & +\frac{1}{24} \beta\left(15 A^{2}-B^{2}+12 A B\right) \\
& -\frac{1}{12} \beta^{2} R^{2}(11 A-3 B)=0 .
\end{aligned}
$$

The bifurcation behavior of the system (2.4) when the resonance condition (3.1) holds can be determined from analysis of the system (3.7). It is important to note that the significant difference between (3.7) and the corresponding system (2.28) is the presence of quadratic nonlinearities in the former. The effects of these will be revealed below. We observe, however, that since small-parameter scaling has already been effected, the quadratic and cubic nonlinearities in (3.7) are of equal importance.

We begin by considering stability. Linearization of (3.7) gives

$$
\begin{gathered}
5 A_{\tau}-\left(\mu+\frac{1}{8}\right) A+\left(5 \gamma-2 \mu+\frac{23}{24}\right) B=0, \\
5 B_{\tau}+\left(2 \mu-5 \gamma+\frac{1}{24}\right) A-\left(\mu-\frac{3}{8}\right) B=0 .
\end{gathered}
$$

Setting $A, B$ proportional to $\exp (\nu \tau)$ we obtain the following characteristic equation for the stability exponents:

$$
5 \nu^{2}-\left(2 \mu-\frac{1}{4}\right) \nu+\Delta=0
$$

where

$$
\Delta \equiv \mu^{2}-\left(4 \gamma+\frac{5}{12}\right) \mu+5 \gamma^{2}+\frac{11}{12} \gamma-\frac{5}{288} .
$$

As expected these equations show that the null solution is stable when $\mu$ is negative and sufficiently large, and is unstable when $\mu$ is positive and sufficiently large. Further investigation reveals that loss of stability of the null solution can occur in essentially one of two possible ways, depending on the value of $\gamma$.

Case 1. We see from (3.9) that loss of stability takes place as $\mu$ increases through the value

$$
\mu=\frac{1}{8}
$$

provided that $\Delta>0$ when $\mu=\frac{1}{8}$. Now

$$
\Delta\left(\mu=\frac{1}{8}\right)=5 \gamma^{2}+\frac{5}{12} \gamma-\frac{31}{576}
$$

and this is positive for values of $\gamma$ defined by

$$
\gamma<\gamma^{*} \text { or } \gamma>\gamma^{* *},
$$


where

$$
\gamma^{*}, \gamma^{* *}=\frac{-5 \mp 6 \sqrt{5}}{120}
$$

When (3.13) holds the real part of complex conjugate roots of (3.9) changes from negative to positive at $\mu=\frac{1}{8}$.

Case 2. When

$$
\gamma^{*}<\gamma<\gamma^{* *}
$$

we see that $\Delta<0$ at $\mu=\frac{1}{8}$ and hence there is no change of stability at this value. In this case change of sign of a root of (3.9) occurs at values of $\mu$ where $\Delta=0$, that is, at points $\mu_{p}, \mu_{Q}$ defined by

$$
\mu_{p}, \mu_{Q}=\frac{1}{2}\left(4 \gamma+\frac{5}{12} \mp \sqrt{D}\right)
$$

where

$$
D=\left(4 \gamma+\frac{5}{12}\right)^{2}-4\left(5 \gamma^{2}+\frac{11}{12} \gamma-\frac{5}{288}\right) \text {. }
$$

It is easy to show that $D>0$ in the range (3.15), so that (3.16) defines real values. Also, it can easily be shown that in this case

$$
\mu_{p}<\frac{1}{8}, \quad \mu_{Q}>\frac{1}{8} .
$$

When (3.15) holds the stability exponents at $\mu_{p}$ are

$$
\nu=0, \quad \frac{1}{5}\left(2 \mu_{p}-\frac{1}{4}\right)
$$

a simple calculation reveals, moreover, that the first stability exponent changes from negative to positive as $\mu$ increases through $\mu_{p}$, while the second exponent is negative in a neighborhood of $\mu_{p}$. Hence we conclude that the null solution loses stability at $\mu=\mu_{p}$, through the change of sign of a single real exponent.

At $\mu=\mu_{Q}$ the stability exponents are

$$
\nu=\frac{1}{5}\left(2 \mu_{Q}-\frac{1}{4}\right), \quad 0 ;
$$

the first exponent is positive in a neighborhood of $\mu_{Q}$, while the second changes from negative to positive as $\mu$ passes through $\mu_{Q}$. The solution is thus unstable on both sides of $\mu_{Q}$.

We turn now to a consideration of bifurcation behavior for the system (3.7).

Case 1. When (3.13) holds the real part of a pair of complex conjugate stability exponents changes sign at $\mu=\frac{1}{8}$. We anticipate, therefore, the bifurcation from $\mu=\frac{1}{8}$ of a solution of (3.7) which is periodic in $\tau$ : in other words, a Hopf bifurcation. We have not thought it necessary to calculate this solution here, since the procedure is standard and straightforward. When $\left|\mu-\frac{1}{8}\right|$ is small a series expansion of the solution can be found by the usual methods. If $\nu_{i}$ is the imaginary part of the stability exponent $\nu$ at $\mu=\frac{1}{8}$, the period of the bifurcating solution will tend to $2 \pi /\left|\nu_{i}\right|$ in $\tau$ as $\left|\mu-\frac{1}{8}\right| \rightarrow 0$. For moderate or large values of $\left|\mu-\frac{1}{8}\right|$ the solutions would have to be calculated numerically.

The fact that the solution of (3.7) is periodic in $\tau$ implies that the solution of the original problem represents a quasiperiodic function. Thus the behavior when (3.13) holds is essentially similar to that discussed in the preceding section for general values of $\Omega$. We may infer, therefore, that when $|\gamma|$ is large enough for (3.13) to hold we are far enough away from resonance conditions, and the closeness of the natural and 
modulating frequencies defined by (3.1) has no special significance. More detailed discussion of this case in a related context can be found in Rosenblat [4].

Case 2. When (3.15) holds a real stability exponent changes sign at both $\mu=\mu_{P}$ and $\mu=\mu_{Q}$. Hence each of $\mu_{P}, \mu_{Q}$ is a point from which a $\tau$-independent bifurcating solution of (3.7) will emerge. Due to the presence of quadratic nonlinearities in (3.7) we expect each of these bifurcating solutions to be transcritical with respect to the points $\mu_{P}, \mu_{Q}$ respectively. The solution from $\mu_{P}$ will be unstable on the subcritical side $\left(\mu<\mu_{P}\right)$ and stable on the supercritical side $\left(\mu>\mu_{P}\right)$, while the solution from $\mu_{Q}$ will be unstable on both sides of $\mu_{Q}$. These remarks are verifiable by appeal to general bifurcation theory or by direct analysis in the neighborhoods of the bifurcation points.

Next, one should ask what happens to these solutions at large distances from $\mu_{P}$, $\mu_{Q}$, in particular whether their norms grow without limit. Solutions of (3.7) which are $\tau$-independent and have large norms are seen to be governed approximately for large $|\mu|$ by the following equations:

$$
\begin{aligned}
& 0=-\mu A-2 \mu B+\frac{1}{12} \beta^{2} R^{2}(3 A+11 B), \\
& 0=2 \mu A-\mu B-\frac{1}{12} \beta^{2} R^{2}(11 A-3 B)
\end{aligned}
$$

These are (3.7) for $\tau$-independent solutions with the quadratic and some other smaller terms omitted. From an examination of these we are quickly led to the conclusion that the system (3.21) admits no real solutions. Hence we can infer that (3.7) has no $\tau$-independent solutions of large norm for large $|\mu|$. An inspection of (3.7) also reveals that there are no $\tau$-independent solutions (other than the trivial) of small norm for large $|\mu|$. On the basis of this analysis, therefore it is tempting to conclude that the $\tau$-independent solutions bifurcating from $\mu_{P}, \mu_{Q}$ terminate at finite values of $\mu$.

To gain further insight into the bifurcation behavior we have computed numerically the $\tau$-independent solutions of (3.7) in the special case $\gamma=0, \beta=1$. (There seems no reason to believe that the results are qualitatively different if $\gamma \neq 0$ or $\beta \neq 1$.) In other words we have calculated the nontrivial real roots of the coupled cubic equations

$$
\begin{aligned}
& 0=-\left(\mu+\frac{1}{8}\right) A-\left(2 \mu-\frac{23}{24}\right) B-\frac{1}{24}\left(15 A^{2}+27 B^{2}+16 A B\right)+\frac{1}{12} R^{2}(3 A+11 B), \\
& 0=\left(2 \mu+\frac{1}{24}\right) A-\left(\mu-\frac{3}{8}\right) B+\frac{1}{24}\left(15 A^{2}-B^{2}+12 A B\right)-\frac{1}{12} R^{2}(11 A-3 B) .
\end{aligned}
$$

The computations were performed on a Cyber digital computer at the University of Melbourne; the solution of the algebraic equations was accomplished using a library subroutine called Calfun.

The results of the calculations are shown in Fig. 1, which depicts the norm $R$ of solutions as a function of $\mu$. We see that two solutions emerge from each of the bifurcation points $P, Q$ (which correspond to the values $\mu_{P}, \mu_{Q}$ ), one supercritical and one subcritical. The supercritical branch $P F$ from $P$ was continued numerically to a value $\mu \approx 1.8$; beyond this value no extension of this solution could be found. Similarly, the supercritical branch $Q F$ from $Q$ could not be continued beyond $\mu \approx 1.8$. Since these two solution curves approached each other in norm there seems little doubt that they actually meet at $F$, and that there is in fact one closed continuous solution branch $P F Q$ joining the two bifurcation points $P, Q$ and supercritical with respect to both of them. In like fashion the subcritical branches beginning at $P$ and $Q$ each appear to turn around, at $G$ and $H$ respectively, and meet at $E$, where $\mu \approx 1.64$, to form a continuous branch $P G E H Q$. A thorough numerical search revealed no nontrivial solution of (3.22) to the right of $F$ or to the left of $G$. 


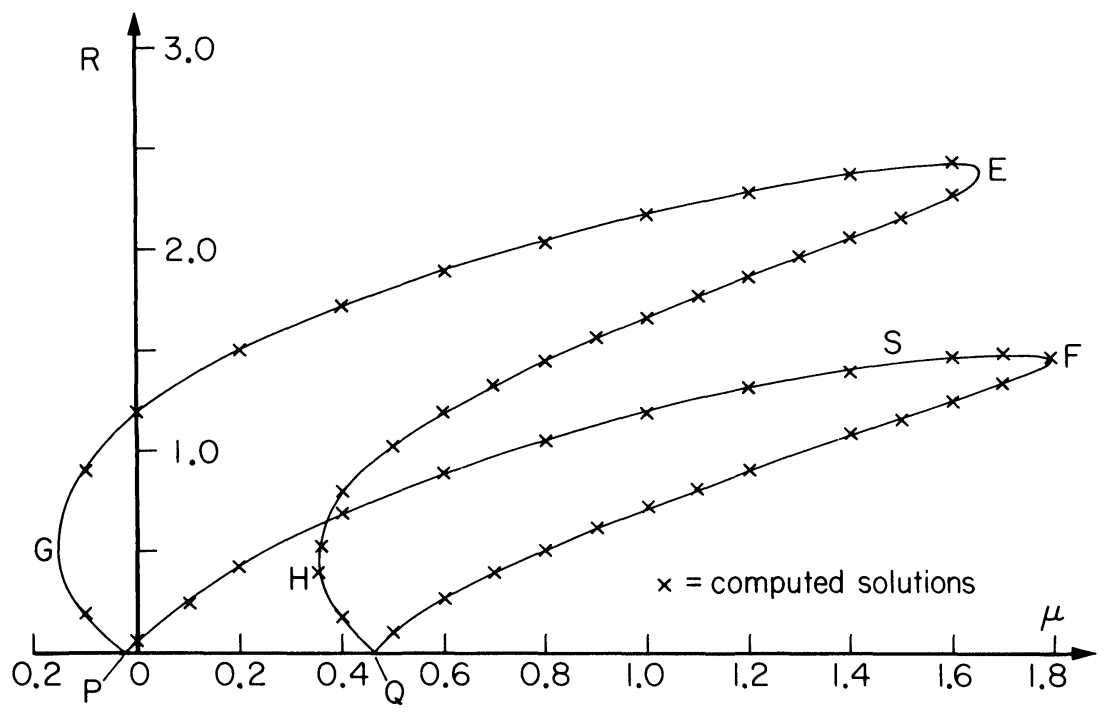

FIG. 1

It would seem, therefore, that when (3.15) holds the system (3.7) admits $\tau$ independent solutions for a range of values of $\mu$, namely

$$
\mu_{G}<\mu<\mu_{F}
$$

When this occurs it is easy to see that the bifurcating solution is periodic, with period $2 \pi$ in the time $t^{*}$; in the natural time $t$ the period is

$$
\frac{2 \pi}{1+\varepsilon^{2} \gamma}=\frac{2 \pi}{\Omega}
$$

This is an example of the well-known phenomenon of frequency locking or frequency entrainment: when the natural and modulating frequencies are close enough the system responds at the modulating frequency.

On the other hand the inequalities (3.23) show that this phenomenon occurs only in a restricted range. Using (2.11) we can replace (3.23) by

$$
2 \varepsilon^{2} \mu_{G}<\alpha-2<2 \varepsilon^{2} \mu_{F}
$$

where from Fig. 1 we have $\mu_{G} \approx-0.16$ and $\mu_{F} \approx 1.80$. This formulation makes it clear that frequency locking is a very localized event.

To determine what happens outside the range (3.23), or (3.25), it is necessary to look at the possibility of new solutions of (3.7) bifurcating from the computed solutions shown in Fig. 1. We have not attempted this investigation here; nevertheless certain conjectures can reasonably be advanced. First of all, we know that any new solution cannot be $\tau$-independent, since otherwise it would have appeared in the computations. Secondly, we know from local analysis that the supercritical solution $P F$ is stable close enough to $P$, while the supercritical solution $Q F$ is unstable close enough to $Q$. Hence, there is a point $S$ somewhere on the supercritical curve $P F Q$ at which the solution changes stability. This point may or may not coincide with the turning point $F$; in either case $S$ is potentially a bifurcation point for a new solution. Since this solution cannot be $\tau$-independent it seems reasonable to suggest that it is $\tau$-periodic and, more 
over, that it exists for values of $\mu$ beyond the terminal value $\mu_{F} \approx 1.80$. Similar behavior has been discussed recently by Rosenblat and Cohen [3] and Kath [2].

What happens on the branch $P G E H Q$ is less clear since this solution is unstable close enough to both $P$ and $Q$; consequently there may be no bifurcation points on this curve, or there may be more than one. This requires further investigation.

If the conjecture stated in the penultimate paragraph is correct, the bifurcation of a $\tau$-periodic solution of (3.7) from the point $S$ implies that the solution of the original problem is quasiperiodic for as long as this branch persists. The solution would then be qualitatively little different from that discussed in $\S 2$; we would infer that frequency locking is strictly a local phenomenon, and that quasiperiodicity is the "natural" state for the bifurcating solutions.

4. Bifurcation when $\Omega \approx 2$. We consider next the situation where the modulating frequency is nearly twice the natural frequency at criticality. The appropriate range for resonance in this case turns out to be given by

$$
\Omega=2(1+\varepsilon \gamma) \text {, }
$$

where $\gamma$ is a constant.

We define time scales $t^{*}, \sigma$ by the formulae

$$
t^{*}=(1+\varepsilon \gamma) t, \quad \sigma=\varepsilon t,
$$

so that the system (2.4) becomes

$$
\begin{aligned}
& (1+\varepsilon \gamma) u_{t^{*}}+\varepsilon u_{\sigma}=-\alpha\left(1+\varepsilon \cos 2 t^{*}\right)(v+\beta u v), \\
& (1+\varepsilon \gamma) v_{t^{*}}+\varepsilon v_{\sigma}=-v+w \\
& (1+\varepsilon \gamma) w_{t^{*}}+\varepsilon w_{\sigma}=u-w
\end{aligned}
$$

In this case we set

$$
\alpha=2(1+\varepsilon \mu+\cdots)
$$

and expand the vector $\mathbf{q}=(u, v, w)$ in the form

$$
\mathbf{q}\left(t^{*}, \sigma\right)=\varepsilon^{1 / 2} \mathbf{q}_{1}\left(t^{*}, \sigma\right)+\varepsilon \mathbf{q}_{2}\left(t^{*}, \sigma\right)+\varepsilon^{3 / 2} \mathbf{q}_{3}\left(t^{*}, \sigma\right)+\cdots
$$

Clearly the response is greater when $\Lambda=2$ than for other values of $\Lambda$.

Substituting (4.4)-(4.5) into (4.3) and equating coefficients of like powers of $\varepsilon$, we obtain a sequence of equations, the first of which is identical with (2.12), and the next two are

$$
\begin{aligned}
& L\left(\mathbf{q}_{2}\right)=\left(-2 \beta u_{1} v_{1}, 0,0\right), \\
& L\left(\mathbf{q}_{3}\right)=-\left(u_{1, \sigma}+\gamma u_{1, t^{*}}+2 \mu v_{1}+Z, v_{1, \sigma}+\gamma v_{1, t^{*}}, w_{1, \sigma}+\gamma w_{1, t^{*}}\right),
\end{aligned}
$$

where $L$ is the operator (2.15) with $t$ replaced by $t^{*}$, and where

$$
Z=2 \beta\left(u_{1} v_{2}+u_{2} v_{1}\right)+2 \cos 2 t^{*} v_{1} \text {. }
$$

The solution for $\mathbf{q}_{1}$ is (2.17) with $\tau$ replaced by $\sigma$ and $t$ by $t^{*}$. Equation (4.6) is identical with (2.19) and can be solved in standard manner. We then calculate $Z$ and apply the Fredholm alternative to (4.8) to obtain, after some simplifying algebra, the following equations for the amplitude functions $A$ and $B$ :

$$
\begin{aligned}
& 5 A_{\sigma}+\left(\frac{1}{2}-\mu\right) A+(5 \gamma-1-2 \mu) B+\frac{1}{12} \beta^{2} R^{2}(3 A+11 B)=0 \\
& 5 B_{\sigma}-(5 \gamma+1-2 \mu) A-\left(\frac{1}{2}+\mu\right) B-\frac{1}{12} \beta^{2} R^{2}(11 A-3 B)=0 .
\end{aligned}
$$


Equations (4.9) describe the bifurcation behavior in the resonance case (4.1). It is interesting to note that, by comparison with the corresponding equations (3.7) in the previous section, (4.9) have no quadratic nonlinearities. In fact the nonlinear terms are identical with those appearing in (2.28), and the systems differ only in the linear terms.

The analysis of (4.9) is very similar to that of (3.7) in the previous section. We begin by considering the stability of the null solution: linearizing (4.9) and setting $A$, $B$ proportional to $\exp (\nu \sigma)$, we obtain the characteristic equation

$$
5 \nu^{2}-2 \mu \nu+\Delta=0
$$

where

$$
\Delta=\mu^{2}-4 \gamma \mu+5 \gamma^{2}-\frac{1}{4} .
$$

As before, two cases can be distinguished.

Case 1. If $\Delta(0)>0$ the null solution loses stability as $\mu$ increases through zero. From (4.11) we see, therefore, that loss of stability occurs at $\mu=0$ whenever

$$
\gamma<-\frac{1}{2 \sqrt{5}} \quad \text { or } \quad \gamma>\frac{1}{2 \sqrt{5}} .
$$

In this case, moreover, the real part of a pair of complex conjugate eigenvalues changes sign at $\mu=0$.

When (4.12) holds, a periodic solution of (4.9) bifurcates from the null solution at $\mu=0$. This solution can be computed in the usual way, and can be shown to be supercritical and stable. The solution has period $2 \pi /\left|\nu_{i}\right|$ in $\sigma$ as $\mu \rightarrow 0$, where $\nu_{i}$ is the imaginary part of the stability exponent at $\mu=0$.

The solution of (4.9) being periodic implies that the bifurcating solution (4.5) is quasiperiodic, in the same way as in $\S 3$. When $|\gamma|$ is large enough that (4.12) holds there is no frequency locking in this case.

Case 2. When

$$
-\frac{1}{2 \sqrt{5}}<\gamma<\frac{1}{2 \sqrt{5}}
$$

we see from (4.11) that $\Delta(0)<0$, but that $\Delta=0$ at real values $\mu_{p}, \mu_{Q}$ given by

$$
\mu_{P}, \mu_{Q}=\frac{1}{2}\left\{4 \gamma \mp \sqrt{1-4 \gamma^{2}}\right\} \text {. }
$$

It is clear from (4.14) that $\mu_{P}<0$ and $\mu_{Q}>0$. At $\mu=\mu_{P}$ the stability exponents are

$$
\nu=0, \quad \frac{2}{5} \mu_{P}
$$

the first exponent goes from negative to positive as $\mu$ increases through 0 , while the second exponent remains negative in a neighborhood of $\mu=0$. It follows that the null solution loses stability at $\mu=\mu_{P}$. The stability exponents at $\mu=\mu_{Q}$ are

$$
\nu=\frac{2}{5} \mu_{Q}, \quad 0
$$

the null solution remains unstable as $\mu$ crosses this value, but the second stability exponent changes from negative to positive.

Each of the points $\mu_{P}, \mu_{Q}$ is a potential bifurcation point for a $\tau$-independent solution of (4.9) bifurcating from the null solution. To determine these steady solutions we set $A_{\sigma}, B_{\sigma}$ equal to zero in (4.9) to obtain a pair of algebraic equations for $A$ and $B$. It is convenient to transform to polar coordinates defined by $(2.30)$. Then the 
$\sigma$-independent equations we seek to solve are found to be

$$
\begin{aligned}
& \mu+\frac{\sqrt{5}}{2} \sin (2 \theta-\phi)-\frac{1}{4} \beta^{2} R^{2}=0, \\
& 5 \gamma-2 \mu+\frac{\sqrt{5}}{2} \cos (2 \theta-\phi)+\frac{11}{12} \beta^{2} R^{2}=0,
\end{aligned}
$$

where $\tan \phi=\frac{1}{2}$.

We can analyze these equations in the following way. Eliminating $\theta$ we obtain a quartic equation for $R$, namely

$$
\frac{13}{72} \beta^{4} R^{4}+\frac{1}{6}(11 \gamma-5 \mu) \beta^{2} R^{2}+\Delta=0,
$$

where $\Delta$ is defined by (4.11). Inspection of (4.18) reveals the following. When $\Delta<0$ there is just one real positive root $R^{2}$ of $(4.18)$. This implies just one real positive root $R$, which in turn is equivalent to a single nontrivial solution of the system (4.17). Now from (4.11) and (4.14) we see that $\Delta<0$ corresponds to values of $\mu$ in the range

$$
\mu_{P}<\mu<\mu_{Q}
$$

We conclude, therefore, that there is just one $\sigma$-independent bifurcating solution when $\mu$ lies in the range (4.19).

Next we see from (4.18) that there are two positive real roots $R^{2}$ when

$$
0<\Delta<\frac{1}{26}(11 \gamma-5 \mu)^{2} \text { and } 5 \mu>11 \gamma .
$$

Elementary calculations show that the inequalities (4.20) are together equivalent to $\mu$ lying in the interval

$$
\mu_{Q}<\mu<\mu_{F}
$$

where

$$
\mu_{F}=-3 \gamma+\frac{1}{2} \sqrt{26}
$$

Thus there are two bifurcating solutions when $\mu$ is in the range (4.21).

Finally, it is easy to see that when

$$
\mu<\mu_{P} \text { and } \mu>\mu_{F}
$$

there are no real roots of (4.18), and hence no $\sigma$-independent bifurcating solutions.

From this discussion we can infer that the bifurcation diagram for $\sigma$-independent solutions of (4.9) is as shown in Fig. 2. (By contrast with Fig. 1 this diagram is schematic, but the picture has been confirmed by selected numerical computations similar to those outlined in $\S 3$.) There is a single continuous branch of solutions joining the points $P, Q$ and extending to the right beyond $Q$ as far as the point $F$. Unlike Fig. 1 there is now only one continuous solution branch. This is due to the absence of quadratic nonlinearities in (4.9), which results in the degeneration and coalescence of the two distinct solution curves of Fig. 1.

Apart from the last-mentioned feature the situation is very similar to that described in $\S 3$. When $\gamma$ lies in the range (4.13) the system (4.9) admits $\sigma$-independent solutions in the range

$$
\mu_{P}<\mu<\mu_{F}
$$




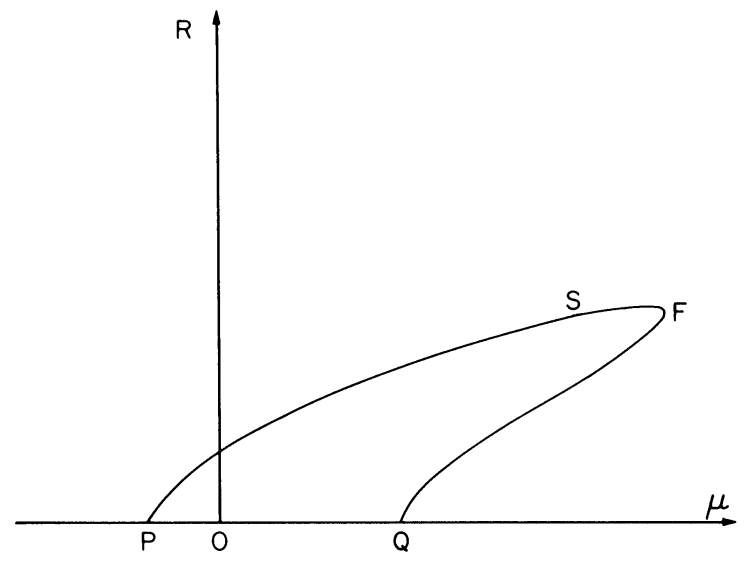

FIG. 2

In this case the bifurcating solution (4.5) is periodic with period

$$
\frac{2 \pi}{1+\varepsilon \gamma}=\frac{4 \pi}{\Omega}
$$

in the natural time $t$. This represents frequency locking due to resonance at the subharmonic of order 2. As before, however, this phenomenon exists only in a local neighborhood of the critical point: the range (4.24) in the natural parameters is

$$
2 \varepsilon \mu_{P}<\alpha-2<2 \varepsilon \mu_{F}
$$

Outside this range we can reasonably expect periodic solutions of (4.9).

The relative simplicity of (4.9) makes it possible to obtain some stability results analytically. The system (4.9) in polar coordinates is

$$
\begin{aligned}
& 5 R_{\sigma}=\mu R+\frac{\sqrt{5}}{2} R \sin (2 \theta-\phi)-\frac{1}{4} \beta^{2} R^{3}, \\
& 5 \theta_{\sigma}=5 \gamma-2 \mu+\frac{\sqrt{5}}{2} \cos (2 \theta-\phi)+\frac{11}{12} \beta^{2} R^{2} .
\end{aligned}
$$

Denoting by $\bar{R}, \bar{\theta}$ a $\sigma$-independent solution of these equations, we investigate its stability by setting

$$
R=\bar{R}+r, \quad \theta=\bar{\theta}+\zeta
$$

in (4.27) and linearizing. This gives

$$
\begin{aligned}
& 5 r_{\sigma}=-\frac{1}{2} \beta^{2} \bar{R}^{2} r-\bar{R}\left(10 \gamma-4 \mu+\frac{11}{6} \beta^{2} \bar{R}^{2}\right) \zeta, \\
& 5 \zeta_{\sigma}=\frac{11}{6} \beta^{2} \bar{R} r+\left(2 \mu-\frac{1}{2} \beta^{2} \bar{R}^{2}\right) \zeta .
\end{aligned}
$$

The stability exponents $\nu$ for this system are found to be the roots of the quadratic equation

$$
5 \nu^{2}-n \nu+\frac{1}{18} \beta^{2} \bar{R}^{2} m=0
$$

where

$$
n=2 \mu-\beta^{2} \bar{R}^{2}, \quad m=13 \beta^{2} \bar{R}^{2}+6(11 \gamma-5 \mu)
$$


From (4.18) it is easily shown that $m<0$ on the lower branch $Q F$ in Fig. 2, and $m>0$ on the upper branch $P F ; m=0$ at $F$. This implies that the solution branch $Q F$ is unstable. The stability of $P F$, on the other hand, is determined by the sign of the quantity $n: P F$ is stable if $n<0$ and is unstable if $n>0$. Since $\mu_{P}<0$ we know that $n<0$ close to the point $P$. Hence the solution along $P F$ is stable close enough to $P$, but it necessarily loses stability at some point $S$ on $P F$. Whether or not $S$ coincides with $F$ can be determined numerically; but we have not performed this calculation. In any case it seems very likely that a new solution bifurcates from the $\sigma$-independent solution at $S$, and that this new solution is periodic in $\sigma$. This eventuality would imply, as in the preceding section, a quasiperiodic solution of (2.4) except in the immediate neighborhood of the critical point $\alpha=2$.

5. Bifurcation when $\Omega \approx 3$. In this case the effective range for possible resonance is given by

$$
\Omega=3\left(1+\varepsilon^{2} \gamma\right)
$$

with $\gamma$ a constant. The solution procedure is very similar to the case $\Omega \approx 1$ considered in $\S 3$, and for this reason we omit many of the details of the calculations.

We use again the times $t^{*}, \tau$ defined by (3.2). The first equation of (2.4) becomes

$$
\left(1+\varepsilon^{2} \gamma\right) u_{t^{*}}+\varepsilon^{2} u_{\tau}=-\alpha\left(1+\varepsilon \cos 3 t^{*}\right)(v+\beta u v),
$$

while the second and third of (2.4) become the second and third of (3.3). The vector $\mathbf{q}$ is expanded in the series (2.10) while we retain the representation (2.11) to define the bifurcation parameter $\mu$.

The usual expansion procedure leads at first order to the system (2.12), with general solution (3.17). At second order we have (2.13) with $\Omega t$ replaced by $3 t^{*}$, while at third order we have (3.5), where $Z$ is given by (2.16) with $\Omega t$ replaced by $3 t^{*}$. Following the same procedure as before we obtain eventually the following equations for the amplitudes $A, B$ :

$$
\begin{aligned}
5 A_{\tau}-\left(\mu-\frac{1}{40}\right) A+\left(5 \gamma-2 \mu-\frac{1}{8}\right) B & +\frac{1}{24} \beta\left(7 A^{2}-7 B^{2}-38 A B\right) \\
& +\frac{1}{12} \beta^{2} R^{2}(3 A+11 B)=0, \\
5 B_{\tau}+\left(2 \mu+\frac{1}{8}-5 \gamma\right) A-\left(\mu-\frac{1}{40}\right) B & +\frac{1}{24} \beta\left(19 B^{2}-19 A^{2}-14 A B\right) \\
& -\frac{1}{12} \beta^{2} R^{2}(11 A-3 B)=0 .
\end{aligned}
$$

These equations are similar to (3.7) in that they contain both cubic and quadratic nonlinearities; there are, however, significant differences, particularly in the structure of the linear terms.

For the stability of the null solution we linearize (5.3) and set $A, B$ proportional to $\exp (\nu \tau)$. The stability exponents are now clearly given by

$$
\nu=\nu_{r} \pm i \nu_{i}=\mu-\frac{1}{40} \pm i\left(2 \mu+\frac{1}{8}-5 \gamma\right) \text {. }
$$

It follows that the null solution loses stability as $\mu$ increases through the value

$$
\mu=\frac{1}{40} \text {, }
$$

independent of the value of $\gamma$. Provided that $\nu_{i} \neq 0$ at $\mu=\frac{1}{40}$ the loss of stability is through the change of sign of the real part of a pair of complex conjugate eigenvalues. Obviously this will be the case for all values of $\gamma$ except

$$
\gamma=\frac{7}{200} \text {. }
$$


When (5.6) holds the stability exponent at the critical point (5.5) is a double real eigenvalue of the relevant stability matrix.

Leaving aside for the moment the special case (5.6) we anticipate in general a Hopf bifurcation from the null solution at $\mu=\frac{1}{40}$. Except when (5.6) holds, therefore, a $\tau$-periodic solution of (5.3) will bifurcate and can be calculated in the usual way. It can be shown that this solution is supercritical and stable.

The periodic bifurcating solution of (5.3) implies a quasiperiodic bifurcating solution of the form (2.10) of the original problem. Thus when (5.1) applies we do not have frequency locking near resonance except perhaps in the special case (5.6).

We turn now to the latter and look for frequency-locked solutions when (5.6) holds; that is, we look for $\tau$-independent solutions of (5.3) when $\gamma$ is given by (5.6). Converting to polar coordinates we see readily that such solutions are solutions of the pair of algebraic equations

$$
\begin{aligned}
& 0=\bar{\mu}-b \beta R \cos (3 \theta+\phi)-\frac{1}{4} \beta^{2} R^{2}, \\
& 0=2 \bar{\mu}-b \beta R \sin (3 \theta+\phi)-\frac{11}{12} \beta^{2} R^{2},
\end{aligned}
$$

where

$$
\bar{\mu}=\mu-\frac{1}{40}, \quad \tan \phi=\frac{19}{7}, \quad b=\frac{\sqrt{410}}{24} .
$$

Eliminating $\theta$ from the equations (5.7) we obtain a quartic equation for $R$, namely

$$
52 \beta^{4} R^{4}-(240 \bar{\mu}+41) \beta^{2} R^{2}+288 \bar{\mu}^{2}=0 .
$$

From the structure of (5.9) it is apparent that there are no real positive roots when $\bar{\mu}<\bar{\mu}_{G}$, where

$$
240 \bar{\mu}_{G}+41=0,
$$

and no real positive roots when $\bar{\mu}>\bar{\mu}_{F}$, where $\bar{\mu}_{F}$ is the positive root of the quadratic

$$
(240 \bar{\mu}+41)^{2}=4 \cdot 52 \cdot 288 \bar{\mu}_{2} .
$$

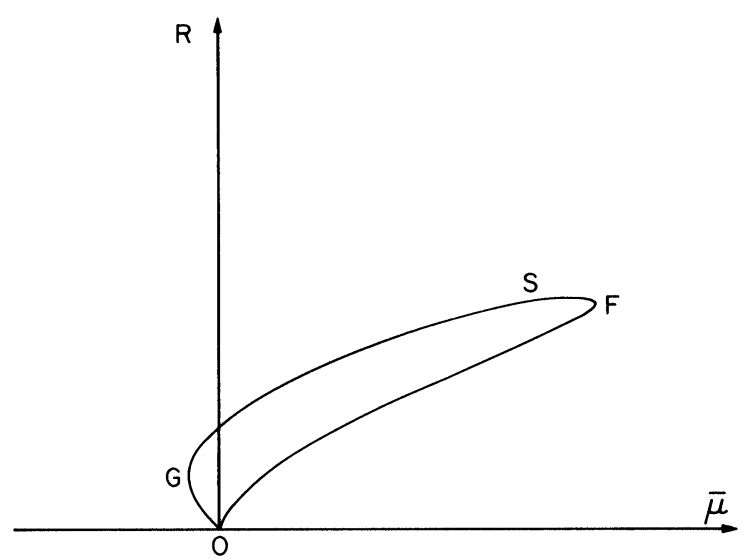

FIG. 3 
In the range

$$
\bar{\mu}_{G}<\bar{\mu}<\bar{\mu}_{F},
$$

on the other hand, there are two positive roots, and it can be inferred that the bifurcation diagram in this case is as shown in Fig. 3.

Once again the phenomenon of frequency locking is completely local, and, moreover, in this case occurs only for the special value (5.6) of $\gamma$. Because of the special nature of this behavior it seems unnecessary to pursue the question of the stability of the solutions of (5.9), particularly since this has been done in slightly different contexts by Rosenblat and Cohen [3] and Rosenblat [4]. As in $\S \S 3$ and 4 it seems reasonable to conjecture that a new $\tau$-periodic solution bifurcates from the solution curve shown in Fig. 3, and corresponds to a quasiperiodic solution of the original problem.

Acknowledgments. The authors are indebted to Dr. P. T. Cummings for writing the computer program used in $\S 4$, and to Dr. J. S. Richardson for help in implementing the program.

\section{REFERENCES}

[1] J. M. Cushing, Integrodifferential Equations and Delay Models in Population Dynamics, Lecture Notes in Biomathematics, 2, Springer-Verlag, Berlin, 1977.

[2] W. KAтH, Resonance in periodically perturbed Hopf bifurcation, Stud. Appl. Math., 65 (1981), pp. 95-112.

[3] S. Rosenblat And D. S. Cohen, Periodically perturbed bifurcation, part 2, Hopf bifurcation, Stud. Appl. Math., 64 (1981), pp. 143-175.

[4] S. Rosenblat, Parametric modulation of a Hopf bifurcation, to appear. 